\title{
Detecting planetary signals with Bayesian methods
}

\author{
Samuli Kotiranta and Mikko Tuomi \\ Department of Physics and Astronomy, University of Turku, Turku, Finland \\ email: samuli.kotiranta@utu.fi
}

\begin{abstract}
In this paper we present an application of Bayesian model comparison to the radial velocity measurements of suspected extra-solar planetary system host star.
\end{abstract}

The Bayesian methods can be seen as an alternative to the classical statistical tools. Although these methods are generally well known, their use in astronomy has been concentrated on few specific areas where parameter space is high dimensional and analysis is difficult to perform through more conventional 'frequentist methods'. However, explicit use of Bayesian tools may reveal important new properties from simpler systems as well.

The inverse solution, of a $k$-planet radial velocity model $r_{i}\left(t_{i}\right)=\left[\sum_{j=1}^{k} K_{j}\left[\cos \left(\nu_{j}(t)+\right.\right.\right.$ $\left.\left.\left.\omega_{j}\right)+e_{j} \cos \omega_{j}\right]\right]_{i}+\gamma_{i}+\epsilon_{I i}+\epsilon_{J i}$ was calculated with Markov chain Monte Carlo (MCMC) with Metropolis-Hastings algorithm (Hastings 1970). Here $r$ is measured value at time $t$ while $K, \nu, \omega$ and $e$ are the radial velocity semi-amplitude, true anomaly, longitude of pericentre and eccentricity, respectively. Parameter $\gamma$ is the reference velocity. The instrument error $\epsilon_{I}$ and intrinsic stellar radial velocity activity ('jitter') $\epsilon_{J}$ are assumed to be normally distributed and jitter is kept unfixed.

The actual procedure consists of comparing $k$-planet model with the given data. Using this process we get marginal integrals that correspond to the probabilities of each model with $k$ companions. When the most probable model has been found as a result, the Markov chains con be used to estimate the joint probability distribution function of the free parameters.

Because each model consist of $k$ non-interacting planets there is a possibility that solution - even though statistically the most probable - appears to be physically unlikely. The dynamical analysis for the solution is done by taking a number of random initial value sets from the probability distributions. These were then integrated numerically with Bulirch-Stoer method (Bulirsch \& Stoer 1966).

As an successful example of this procedure we found evidence of a new planet, namely HD 11506 c (Tuomi \& Kotiranta 2009).

\section{Acknowledgements}

S. K. acknowledges the IAU and the Magnus Ehrnrooth Foundation for travel grants that made it possible to participate in the IAU XXVIIth General Assembly.

\section{References}

Bulirsch, R. \& Stoer, J. 1966, Numer. Math. 8, 1

Hastings, W. 1970, Biometrika 57, 97

Tuomi, M. \& Kotiranta, S. 2009, A\&A 496, L13 